Energy relaxation probed by weak antilocalization measurements in GaN heterostructures

H. Cheng, N. Biyikli, J. Xie, Ç. Kurdak, and H. Morkoç

Citation: Journal of Applied Physics 106, 103702 (2009);

View online: https://doi.org/10.1063/1.3253746

View Table of Contents: http://aip.scitation.org/toc/jap/106/10

Published by the American Institute of Physics

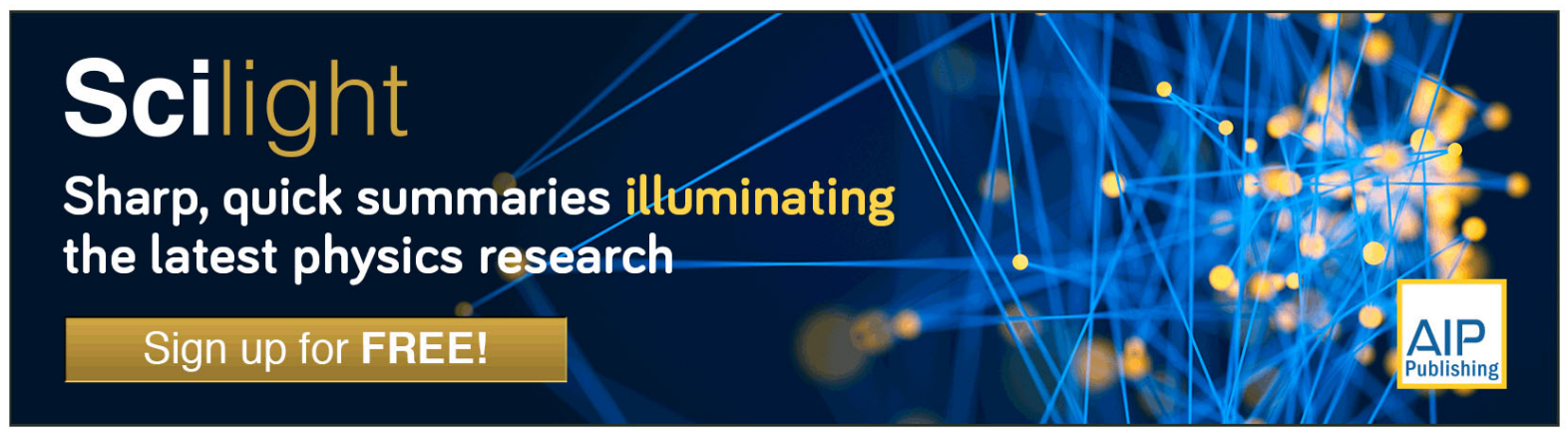




\title{
Energy relaxation probed by weak antilocalization measurements in GaN heterostructures
}

\author{
H. Cheng, ${ }^{1, a)}$ N. Biyikli, ${ }^{2,3}$ J. Xie, ${ }^{2}$ Ç. Kurdak, ${ }^{1}$ and H. Morkoç ${ }^{2}$ \\ ${ }^{1}$ Department of Physics, Randall Lab, University of Michigan, Ann Arbor, Michigan 48109, USA \\ ${ }^{2}$ Department of Electrical Engineering, Virginia Commonwealth University, Richmond, Virginia 23284, USA \\ ${ }^{3}$ UNAM-Institute of Materials Science and Nanotechnology, Bilkent University, Bilkent, Ankara 06800, \\ Turkey
}

(Received 2 March 2009; accepted 29 September 2009; published online 16 November 2009)

Energy relaxation and electron-phonon (e-p) interaction are investigated in wurtzite $\mathrm{Al}_{0.15} \mathrm{Ga}_{0.85} \mathrm{~N} / \mathrm{AlN} / \mathrm{GaN}$ and $\mathrm{Al}_{0.83} \mathrm{In}_{0.17} \mathrm{~N} / \mathrm{AlN} / \mathrm{GaN}$ heterostructures with polarization induced two-dimensional electron gases in the Bloch-Grüneisen regime. Weak antilocalization (WAL) and Shubnikov-de Haas measurements were performed on gated Hall bar structures at temperatures down to $0.3 \mathrm{~K}$. We used WAL as a thermometer to measure the electron temperature $T_{e}$ as a function of the dc bias current. We found that the power dissipated per electron, $P_{e}$, was proportional to $T_{e}^{4}$ due to piezoelectric acoustic phonon emission by hot electrons. We calculated $P_{e}$ as a function of $T_{e}$ without any adjustable parameters for both the static and the dynamic screening cases of piezoelectric e-p coupling. In the temperature range of this experiment, the static screening case was expected to be applicable; however, our data was in better agreement with the dynamic screening case. (C) 2009 American Institute of Physics. [doi:10.1063/1.3253746]

$\mathrm{GaN}$ based semiconductor systems have emerged as potential candidates for a broad range of applications in high power electronics, optoelectronics, and spintronics. ${ }^{1-3} \mathrm{Im}$ proving the $\mathrm{GaN}$ systems' material properties, as well as understanding the fundamental electron transport properties in GaN semiconductor systems, is crucially important in achieving high level device performance. Many of the devices were operated at high bias voltages such that the electrons would equilibrate at a much higher temperature than the lattice temperature. The temperature of those hot electrons was determined by the emission rate of phonons. The study of electron-phonon (e-p) interaction processes is particularly important in this context.

Recent experiments have used noise measurement, Shubnikov-de Hass (SdH) effect, or sample resistivity as thermometers in $\mathrm{GaN}$ two-dimensional electron gas (2DEG) systems to probe the e-p interactions. ${ }^{4-8}$ However, those thermometers have their own limitations. As a complementary method, in this experiment, we utilized the weak antilocalization (WAL) effect as a thermometer at very low temperatures to study e-p interactions in $\mathrm{GaN}$ heterostructures.

It is well known that the WAL arises from quantum interference of spin-dephased electrons and that the spin dephasing process is temperature sensitive. In our previous work, we have used WAL measurements to extract the spinorbit splitting parameter in the GaN systems. ${ }^{9,10}$ From the experiment, we found the size of the WAL feature showed strong temperature dependence and could be utilized as a thermometer to study energy relaxation processes of the 2DEG system.

In this work, we measured two $\mathrm{GaN}$ heterostructures with different barriers. ${ }^{11}$ Both heterostructures were grown

\footnotetext{
a) Author to whom correspondence should be addressed. Tel.: +1-734-6150195. FAX: +1-734-763-9694. Electronic mail: hailing_cheng@hotmail.com.
}

by metalorganic vapor phase epitaxy on $c$-plane sapphire substrates. Sample A has a $25 \mathrm{~nm} \mathrm{Al}_{0.15} \mathrm{Ga}_{0.85} \mathrm{~N}$ barrier layer and sample $\mathrm{B}$ has a $20 \mathrm{~nm} \mathrm{Al} \mathrm{l}_{0.83} \mathrm{In}_{0.17} \mathrm{~N}$ barrier, and both were grown with a $\sim 3-4 \mu \mathrm{m} \mathrm{GaN}$ buffer layer and capped with $\sim 2 \mathrm{~nm} \mathrm{GaN}$. To enhance the electron mobility at low temperature, a thin AlN spacer $(\sim 1 \mathrm{~nm})$ was included between GaN channel and the barrier in both samples. In both heterostructures, all layers were undoped and each 2DEG was formed just below the AlN spacer by spontaneous and piezoelectric polarization effects. To study the magnetotransport properties, $600 \times 100 \mu \mathrm{m}^{2}$ Hall bar structures were fabricated by photolithography followed by dry etching. Ti/Al/ $\mathrm{Ti} / \mathrm{Au}$ contacts annealed at $900{ }^{\circ} \mathrm{C}$ were then used to form Ohmic contacts to the 2DEG. In addition, a Ni/Au gate was deposited on top of sample A to modulate the electron density of the 2DEG.

The samples were characterized by longitudinal magnetoresistance and Hall measurements in two low-temperature cryostats with base temperatures of 0.28 and $1.5 \mathrm{~K}$, respectively. In Fig. 1(a), we plot low temperature magnetoresistance traces for sample A at different gate voltages. This sample exhibited clear $\mathrm{SdH}$ oscillations at $0.28 \mathrm{~K}$. The carrier density extracted from the $\mathrm{SdH}$ oscillations, as well as Hall slopes (not shown here), is found to vary with gate voltages, consistent with a simple capacitance model. For gate voltages of -4 and $4 \mathrm{~V}$, the carrier densities were $3.41 \times 10^{12} \mathrm{~cm}^{-2}$ and $4.92 \times 10^{12} \mathrm{~cm}^{-2}$ and the corresponding mobilities were $8.5 \times 10^{3} \mathrm{~cm}^{2} / \mathrm{V} \mathrm{s}$ and 10.7 $\times 10^{3} \mathrm{~cm}^{2} / \mathrm{V} \mathrm{s}$, respectively. Magnetoresistance and Hall traces for sample B are shown in Fig. 1(b). Unlike sample A, the $\mathrm{SdH}$ oscillations were not well pronounced for sample B. However, after we plot differential resistance in the $\mathrm{SdH}$ data of sample B, clear $\mathrm{SdH}$ oscillations were seen, as shown in the inset of Fig. 1(b). These relatively small SdH oscillations were most likely due to the small spread of nonuniformity of carrier density in sample B. The onset of $\mathrm{SdH}$ oscillations for 


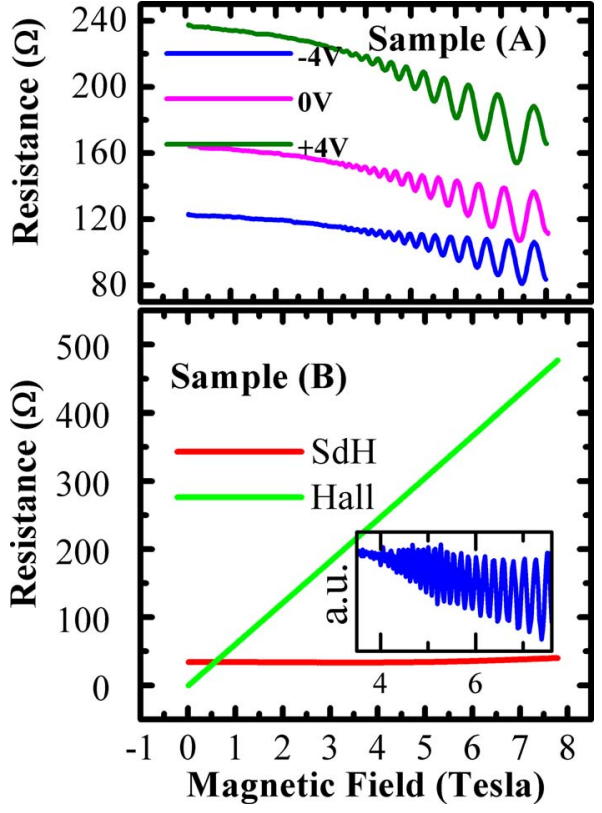

FIG. 1. (Color online) (a) Sample A: Longitudinal resistance measurements at gate voltages from -4 to $4 \mathrm{~V}$ at $0.28 \mathrm{~K}$, with clear $\mathrm{SdH}$ oscillations shown. (b) Sample B: Longitudinal and Hall measurements at $1.5 \mathrm{~K}$. The inset is the differential resistance with clear $\mathrm{SdH}$ oscillations shown.

samples A and B were 3 and 3.5 T, respectively. For sample $\mathrm{B}$, the carrier density was $10.26 \times 10^{12} \mathrm{~cm}^{-2}$ with mobility $17.8 \times 10^{3} \mathrm{~cm}^{2} / \mathrm{V} \mathrm{s}$ at $1.5 \mathrm{~K}$.

We measured the temperature dependence of the quantum corrections to conductance at low magnetic fields using standard four-terminal ac lock-in techniques. Representative traces of magnetoconductivity after subtraction of the zero field background, $\Delta \sigma=\sigma(B)-\sigma(0)$, obtained from sample A with gate voltage $V_{g}=0 \mathrm{~V}$ at different temperatures are shown in Fig. 2(a). There was a clear WAL behavior at magnetic fields below $2 \mathrm{mT}$ from 0.28 to $10 \mathrm{~K}$. This feature arises from the quantum interference of spin-dephased paths
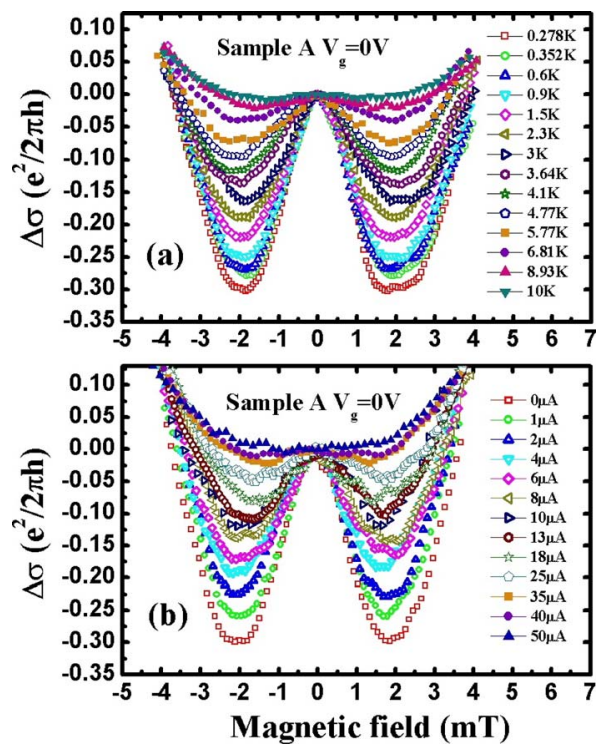

FIG. 2. (Color online) Experimental magnetoconductivity $\Delta \sigma=\sigma(B)-\sigma(0)$ of sample A with $V_{g}=0 \mathrm{~V}$ (a) at different temperatures and (b) at different bias dc currents at $0.28 \mathrm{~K}$.

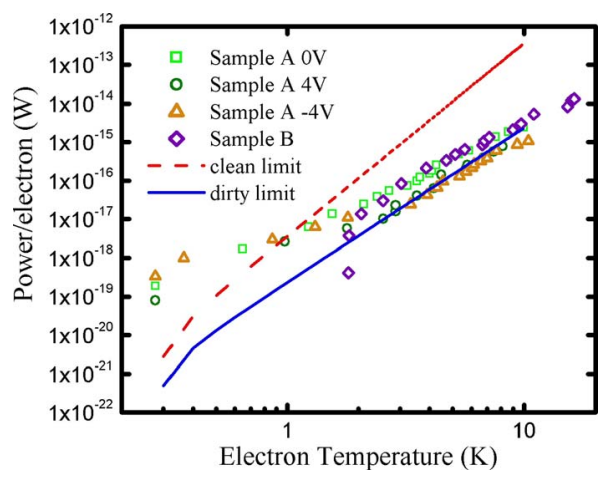

FIG. 3. (Color online) Power dissipated per electron vs electron temperature for samples A and B. The solid line is the calculation for the dirty limit and the dash line is the calculation for the clean limit.

and can be used to quantify spin-orbit coupling in semiconductors. It is obvious in our data that the size of the WAL feature is strongly temperature dependent and decreases with increasing temperature whereas the width of the peak does not vary with temperature. ${ }^{9}$

For ac excitation currents in the range of 10-100 nA, there is no noticeable change in the size of WAL feature. It means that when the system is at such small excitation currents, there is no significant heating for electrons; the electron temperature, $T_{e}$, is close to the lattice temperature $T_{l}$. However, if we pass larger dc bias currents, the electron system would be hotter and the WAL feature would be suppressed. In order to study this heating effect, we kept the sample at the base temperature of the cryostat and passed a dc bias current and a small ac modulation current through the Hall bar structures. The magnetoresistance traces were again measured using the ac lock-in technique. In Fig. 2(b), we plot typical magnetoconductance traces for the sample A at different dc bias currents. We find that the WAL feature decreases with increasing dc bias current.

There is a striking similarity between the traces obtained at higher temperatures and those obtained with higher dc bias currents (Fig. 2). By comparing such sets of WAL traces, we could extract the electron temperature, $T_{e}$, for different bias currents. Instead of plotting electron temperature as a function of bias current, we calculated the power dissipated per electron, $P_{e}$, in the active region of the Hall bar by using equation $P_{e}=I^{2} R /(w L n)$, where $I$ is the device current, $R$ is the four terminal resistance of the Hall bar, $n$ is the two dimensional carrier density, and $w$ and $L$ are the width and length of the Hall bar structure. We plotted the power dissipated per electron versus $T_{e}$ for both sample A at gate voltages $-4,0$, and $4 \mathrm{~V}$ and sample $\mathrm{B}$ in Fig. 3. Plotted in this manner, the heating curves do not depend strongly on the carrier density or the mobility of 2DEG. We note that sample A was measured at a base temperature of $0.28 \mathrm{~K}$ and sample $\mathrm{B}$ was measured at a base temperature of $1.5 \mathrm{~K}$, thus the data obtained from sample A covered a wider temperature range.

In the low-temperature region, the samples are in the Bloch-Grüneisen (B-G) regime, where the average phonon wave vector, $q\left(\sim k_{B} T / \hbar v_{s}\right)$, is much smaller than Fermi wave vector, $k_{F}\left(q \ll k_{F}\right)$. In the B-G regime, the electron energy is not high enough to excite optical phonons; there- 
fore, the dominating power dissipation in the 2DEG system is through the emission of acoustic phonons via the piezoelectric and deformation potential coupling processes. In the B-G regime, the e-p interaction is expected to have various power-law dependences on temperature. ${ }^{5-8,12-14}$ For example, the phonon emission via piezoelectric coupling leads to $T_{e}^{5}$ and $T_{e}^{3}$ dependence for energy relaxation with and without static screening, and the phonon emission via deformation potential leads to $T_{e}^{7}$ and $T_{e}^{5}$ dependence with and without static screening. ${ }^{15}$

At low temperature, it is believed that the piezoelectric coupling is the dominating energy relaxation mechanism in the GaN system. ${ }^{5-8}$ The electron screening effect of local potential must be included in the calculations of energy relaxation rates. At very low temperature, when the wavelength of the emitted phonons becomes comparable to the mean free path of the electrons, the static screening is no longer suitable to describe the system. Instead, dynamic screening should be applied to calculate the piezoelectric coupling case, and as a result the energy relaxation scales as $P_{e}-T_{e}^{4}$. This regime, $q l_{e}<1$, is known as the hydrostatic regime, where $q$ is the average phonon wave vector, $v_{s}$ is the sound velocity, and $l_{e}$ is the elastic mean free path in the material. ${ }^{13}$ This regime is also called the "dirty limit," which is the case of relatively lower mobility systems and has been studied in GaAs 2DEGs ${ }^{12-14}$ before.

The theory for electron energy relaxation in GaAs (zincblende) 2DEG systems both in the dirty limit and in the clean limit has been given in Refs. 14 and 15. However, for wurzite GaN 2DEG systems, the piezoelectric coupling is different from the zincblende type. In wurzite crystals, the e-p matrix elements for longitudinal acoustic (LA) and transverse acoustic (TA) piezoelectric coupling are ${ }^{16}$

$$
\left|M_{\mathrm{LA}}^{\mathrm{PZ}}(\vec{q})\right|^{2}=\frac{\hbar e^{2}}{2 \rho v_{l} q}\left(\frac{e_{33} q_{\perp}^{3}+\left(e_{31}+2 e_{15}\right) q_{\perp} q_{\|}^{2}}{q^{3} \varepsilon}\right)^{2}
$$

and

$$
\left|M_{\mathrm{TA}}^{\mathrm{PZ}}(\vec{q})\right|^{2}=\frac{\hbar e^{2}}{2 \rho v_{t} q}\left(\frac{-\left(e_{33}-e_{31}-e_{15}\right) q_{\perp}^{2} q_{\|}-e_{15} q_{\|}^{3}}{q^{3} \varepsilon}\right)^{2},
$$

respectively. In the above equations, $e_{33}, e_{31}$, and $e_{15}$ are piezoelectric coefficients, $q$ is the magnitude of the threedimensional phonon wave vector, $q_{\perp}$ and $q_{\|}$are the components of $q$ perpendicular and parallel to the 2DEG plane, respectively, $e$ is the electron charge, $\rho$ is the mass density of $\mathrm{GaN}, \varepsilon$ is the dielectric constant in $\mathrm{GaN}$, and $v_{l}$ and $v_{t}$ are LA and TA phonon sound velocities in GaN, respectively.

In the clean limit, we follow the procedures for GaAs reported by Ma et al. ${ }^{15}$ and obtain the piezoelectric LA and TA e-p interaction characteristic function in $\mathrm{GaN}$ as

$$
\begin{aligned}
F_{\mathrm{LA}}^{C}(T)= & \frac{3 \zeta(5) m^{* 2} e^{2}}{32 \pi \hbar^{7} k_{F}^{3} \rho v_{l}^{4} \varepsilon^{2} q_{s}^{2}}\left[5 e_{33}^{2}+6 e_{33}\left(e_{31}+2 e_{15}\right)\right. \\
& \left.+5\left(e_{31}+2 e_{15}\right)^{2}\right]\left(k_{B} T\right)^{5}
\end{aligned}
$$

and

$$
\begin{aligned}
F_{\mathrm{TA}}^{C}(T)= & \frac{3 \zeta(5) m^{* 2} e^{2}}{32 \pi \hbar^{7} k_{F}^{3} \rho v_{l}^{4} \varepsilon^{2} q_{s}^{2}}\left[35 e_{15}^{2}+10 e_{15}\left(e_{33}-e_{31}-e_{15}\right)\right. \\
& \left.+3\left(e_{33}-e_{31}-e_{15}\right)^{2}\right]\left(k_{B} T\right)^{5},
\end{aligned}
$$

respectively, where $\zeta(5)$ is the Riemann zeta function, $m^{*}$ is the effective mass, $k_{F}$ is Fermi wave vector and $q_{s}$ $=m^{*} e^{2} /\left(2 \pi \varepsilon \hbar^{2}\right)$ is the screening wave vector.

In the dirty limit, we follow the procedure given by Chow et al. ${ }^{14}$ and obtain the piezoelectric LA and TA e-p interaction characteristic function in $\mathrm{GaN}$ as

$$
\begin{aligned}
F_{\mathrm{LA}}^{D}(T)= & \frac{\pi^{2}}{15 n \hbar^{3} \sigma_{x x} \rho v_{l}^{3}} \frac{2}{105}\left[15 e_{33}^{2}+12 e_{33}\left(e_{31}+2 e_{15}\right)\right. \\
& \left.+8\left(e_{31}+2 e_{15}\right)^{2}\right]\left(k_{B} T\right)^{4}
\end{aligned}
$$

and

$$
\begin{aligned}
F_{\mathrm{LA}}^{D}(T)= & \frac{\pi^{2}}{15 n \hbar^{3} \sigma_{x x} \rho v_{t}^{3}} \frac{4}{105}\left[24 e_{15}^{2}+8 e_{15}\left(e_{33}-e_{31}-e_{15}\right)\right. \\
& \left.+3\left(e_{33}-e_{31}-e_{15}\right)^{2}\right]\left(k_{B} T\right)^{4},
\end{aligned}
$$

respectively, where $\sigma_{x x}$ is the longitudinal conductivity and $n$ is the carrier density.

The power relaxation rate is $P_{e}=\Sigma_{\lambda} F_{\lambda}\left(T_{e}\right)-F_{\lambda}\left(T_{l}\right)$ with $\lambda$ summed over all phonon modes. In piezoelectric coupling, only two phonon modes should be counted in. ${ }^{16}$ We performed this calculation for all of our samples. When plotted in a logarithmic scale, the calculated $P_{e}$ for our samples did not show obvious difference from each other. Therefore, for the purpose of clarity, we only show a representative calculation for our lowest mobility sample in the clean and dirty limit in Fig. 3. The following values were used in the calculations: $n=3.41 \times 10^{12} \mathrm{~cm}^{-2}, m^{*}=0.21 \mathrm{~m}_{\mathrm{e}}, \rho=6150 \mathrm{~kg} / \mathrm{m}^{3}$, $\varepsilon=10 \varepsilon_{0}, \quad v_{l}=6560 \mathrm{~m} / \mathrm{s}, \quad v_{t}=2680 / \mathrm{s}, \quad \sigma_{x x}=0.00468 / \Omega, e_{15}$ $=-0.3 \mathrm{C} / \mathrm{m}^{2}, e_{31}=-0.49 \mathrm{C} / \mathrm{m}^{2}$, and $e_{33}=0.73 \mathrm{C} / \mathrm{m}^{2}$. ${ }^{7}, 17 \mathrm{It}$ is important to emphasize that there are absolutely no fitting parameters in our calculations.

When comparing our results with the previous reports of energy relaxation by hot electrons, ${ }^{5-8}$ we find that our power dissipation rate per electron is one or two orders of magnitude below those results obtained by using $\mathrm{SdH}$ as a thermometer. When we compare our experimental data with theory, a better agreement with the dirty limit calculation was achieved; the power dissipation rate per electron scales as $P_{e}-T_{e}^{4}$. This dirty limit effect has also been observed in low mobility GaN 2DEG samples. ${ }^{5,8}$ However, our electron mobilities are sufficiently high such that according to $q l_{e}$ criteria, our samples should be in the clean limit where a $P_{e}-T_{e}^{5}$ should be expected. This is contradictory to what we have observed.

In addition to energy relaxation by e-p coupling, other effects such as thermal boundary resistance can also play a significant role in determining the temperature of hot electrons. Based on noise measurements on GaN films grown on sapphire substrates, we have previously observed a large deviation between the measured and calculated electron temperatures from which we extracted a thermal boundary resistance for such an interface. ${ }^{18}$ However, for the 2DEG samples, the total power dissipation rate is small enough that 
we estimate at high bias conditions, when the electron temperature is above $10 \mathrm{~K}$, the contribution due to the thermal boundary resistance is only about $0.01 \mathrm{~K}$. Thus, the thermal boundary cannot explain the observed discrepancy between the measurement and theory.

In summary, we used WAL as a thermometer to measure the electron temperature, $T_{e}$, as a function of the bias current in wurtzite $\mathrm{Al}_{0.15} \mathrm{Ga}_{0.85} \mathrm{~N} / \mathrm{AlN} / \mathrm{GaN}$ and $\mathrm{Al}_{0.83} \mathrm{In}_{0.17} \mathrm{~N} / \mathrm{AlN} /$ $\mathrm{GaN}$ heterostructures with polarization induced $2 \mathrm{DEG}$ in the $\mathrm{B}-\mathrm{R}$ regime. We find that the power dissipated rate per electron, $P_{e}$, is proportional to $T_{e}^{4}$ due to piezoelectric acoustic phonon emission by hot electrons. We calculated power dissipated per electron, $P_{e}$, as a function of $T_{e}$ without using any adjustable parameters for both static and dynamic screening cases of piezoelectric e-p coupling. In the temperature range of this experiment, the static screening mechanism is expected to be applicable; however, our data are in better agreement with the dynamic screening mechanism.

This work is supported by grants from the Air Force Office of Scientific Research (AFOSR) through the Grant No. FA9550-09-0447 under the direction of Dr. G. L. Witt and Dr. K. Reinhardt, and also by the NSF through Grant No. DMR-0606039.

${ }^{1}$ H. Morkoç, Handbook of Nitride Semiconductors and Devices (WileyVCH, Berlin, 2008).
${ }^{2}$ B. Monemar, J. Mater. Sci. Mater. Electron. 10, 227 (1999).

${ }^{3}$ S. Strite and H. Morkoç, J. Vac. Sci. Technol. B 10, 1237 (1992).

${ }^{4}$ M. Ramonas, A. Matulionis, J. Liberis, L. Eastman, X. Chen, and Y. J. Sun, Phys. Rev. B 71, 075324 (2005).

${ }^{5}$ C. E. Martinez, N. M. Stanton, A. J. Kent, M. L. Williams, I. Harrison, H. Tang, J. B. Webb, and J. A. Bardwell, Semicond. Sci. Technol. 21, 1580 (2006).

${ }^{6}$ K. J. Lee, J. J. Harris, A. J. Kent, T. Wang, S. Sakai, D. K. Maude, and J. C. Portal, Appl. Phys. Lett. 78, 2893 (2001).

${ }^{7}$ N. M. Stanton, A. J. Kent, S. A. Cavill, A. V. Akimov, K. J. Lee, J. J. Harris, T. Wang, and S. Sakai, Phys. Status Solidi B 228, 607 (2001).

${ }^{8}$ P. Hawker, A. J. Kent, T. S. Cheng, and C. T. Foxon, Physica B 263-264, 227 (1999)

${ }^{9}$ Ç. Kurdak, N. Biyikli, Ü. Özgür, H. Morkoç, and V. I. Litvinov, Phys. Rev. B 74, 113308 (2006).

${ }^{10}$ Ç. Kurdak, H. Cheng, N. Biyikli, Ü. Özgür, H. Morkoç, and V. I. Litvinov, Proc. SPIE 6894, 68940M (2008).

${ }^{11}$ J. Xie, X. Ni, M. Wu, J. H. Leach, Ü. Özgür, and H. Morkoç, Appl. Phys. Lett. 91, 132116 (2007).

${ }^{12}$ R. Fletcher, Y. Feng, C. T. Foxon, and J. J. Harris, Phys. Rev. B 61, 2028 (2000).

${ }^{13}$ E. Chow, H. P. Wei, S. M. Girvin, W. Jan, and J. E. Cunningham, Phys. Rev. B 56, R1676 (1997).

${ }^{14}$ E. Chow, H. P. Wei, S. M. Girvin, and M. Shayegan, Phys. Rev. Lett. 77, 1143 (1996).

${ }^{15}$ Y. Ma, R. Fletcher, E. Zaremba, M. D’Iorio, C. T. Foxon, and J. J. Harris, Phys. Rev. B 43, 9033 (1991).

${ }^{16}$ P. Tripathi and B. K. Ridley, Phys. Rev. B 66, 195301 (2002).

${ }^{17}$ F. Bernardini, V. Fiorentini, and D. Vanderbilt, Phys. Rev. B 56, R10024 (1997).

${ }^{18}$ T. A. Eckhause, Ö. Süzer, Ç. Kurdak, F. Yun, and H. Morkoç, Appl. Phys. Lett. 82, 3035 (2003). 